\title{
On the Variable Sum Exdeg Index/Coindex of Graphs
}

\author{
Akbar Ali ${ }^{a, 1}$, Emina Milovanović ${ }^{b}$, Marjan Matejić ${ }^{b}$, \\ Igor Milovanović ${ }^{b}$ \\ ${ }^{a}$ Department of Mathematics, Faculty of Science, \\ University of Ha'il, Ha'il, Saudi Arabia \\ akbarali .maths@gmail.com \\ ${ }^{b}$ Faculty of Electronic Engineering, \\ University of Nǐ̌, Nǐ̌, Serbia \\ ema@elfak.ni.ac.rs, marjan.matejic@elfak.ni.ac.rs, \\ igor.milovanovic@elfak.ni.ac.rs
}

(Received November 16, 2020)

\begin{abstract}
Let $G$ be a connected graph with the vertex set $V=\left\{v_{1}, v_{2}, \ldots, v_{n}\right\}$, where $n \geq 2$. Denote by $d_{i}$ the degree of the vertex $v_{i}$ for $i=1,2, \ldots, n$. If $v_{i}$ and $v_{j}$ are adjacent in $G$, we write $i \sim j$, otherwise we write $i \nsim j$. The variable sum exdeg index and coindex of $G$ are defined as $S E I_{a}(G)=\sum_{i \sim j}\left(a^{d_{i}}+a^{d_{j}}\right)=\sum_{i=1}^{n} d_{i} a^{d_{i}}$ and $\overline{S E I}_{a}(G)=\sum_{i \nsim j}\left(a^{d_{i}}+a^{d_{j}}\right)=\sum_{i=1}^{n}\left(n-1-d_{i}\right) a^{d_{i}}$, respectively, where ' $a$ ' is a positive real number different from 1. Some inequalities involving $S E I_{a}(G)$ or/and $\overline{S E I}_{a}(G)$ are derived. Special cases of the obtained inequalities are also discussed for unicyclic graphs.
\end{abstract}

\section{Introduction}

All the graphs discussed in the present paper are simple and connected. Let $G$ be a graph with the vertex set $V=\left\{v_{1}, v_{2}, \ldots, v_{n}\right\}$, edge set $E$ and with the vertex-degree sequence $\left(d_{1}, d_{2}, \cdots, d_{n}\right)$ satisfying $\Delta=d_{1} \geq d_{2} \geq \cdots \geq d_{n}=\delta>0$, where $n \geq 2,|E|=m$ and $d_{i}$ is the degree of the vertex $v_{i}$ for $i=1,2, \cdots, n$. If $v_{i}$ and $v_{j}$ are adjacent in $G$, we write $i \sim j$, otherwise we write $i \nsim j$. Denote by $\bar{G}$ the complement of $G$ and let

\footnotetext{
${ }^{1}$ Corresponding author
} 
$D=\operatorname{diag}\left(d_{1}, d_{2}, \ldots, d_{n}\right)$ be the diagonal degree matrix of $G$. The cyclomatic number of $G$ is the minimum number of edges whose removal from $G$ gives a graph without containing any cycle. Graphs with the cyclomatic number 1, 2, 3 and 4 are usually called unicyclic, bicyclic, tricyclic and tetracyclic graphs, respectively.

The variable sum exdeg index of $G$ is denoted by $S E I_{a}(G)$ and is defined [19] as

$$
S E I_{a}(G)=\sum_{i=1}^{n} d_{i} a^{d_{i}}=\sum_{i \sim j}\left(a^{d_{i}}+a^{d_{j}}\right),
$$

where ' $a$ ' is an arbitrary positive real number different from 1 . The main motivation of introducing and studying the variable sum exdeg index $S E I_{a}$ was its very good chemical applicability - see [19] for detail. Because of its chemical applications, mathematical properties of $S E I_{a}$ have been studied in a considerable number of papers. Vukičević [20] initiated the mathematical study of $S E I_{a}$ and he reported several extremal results concerning $S E I_{a}$. For $a>1$, Ghalavand and Ashrafi [8] determined the graphs having extremum values of $S E I_{a}$ from the classes of all trees and unicyclic graphs of a fixed order. The graphs with the maximum $S E I_{a}$ value (for $a>1$ ) in the classes of all bicyclic and tricyclic graphs of a fixed order were also characterized in [8]. Ali and Dimitrov [1] reported alternative proofs of some of the main results given in [8], and also they proved an extremal result regarding the variable sum exdeg index of tetracyclic graphs. For $a>1$, the results of $[1,8]$ were generalized by Dimitrov and Ali [5] by considering the graphs of a fixed order and cyclomatic number; in [5], the case $0<a<1$ was also discussed but only the partial solutions to the considered problems for this certain case were obtained. The problem of finding graphs having the extremum values of the variable sum exdeg index of the trees of a fixed order and with the vertices having prescribed degrees was attacked in [11]. Additional recent results about the variable sum exdeg index can be found in the papers $[3,7,9,13,18]$.

Motivated from the concept of the Zagreb coindices [6], we define the variable sum exdeg coindex [13] as

$$
\overline{S E I}_{a}(G)=\sum_{i \nsim j}\left(a^{d_{i}}+a^{d_{j}}\right)
$$

which can be rewritten as

$$
\overline{S E I}_{a}(G)=\sum_{i=1}^{n}\left(n-1-d_{i}\right) a^{d_{i}} .
$$

The main purpose of this paper is to derive some inequalities for $S E I_{a}(G)$ or/and $\overline{S E I}_{a}(G)$. Special cases of the obtained inequalities are also considered when $G$ is a unicyclic graph. 


\section{Preliminaries}

In this section, we recall some analytical inequalities for real number sequences that will be used later in the paper.

Let $a=\left(a_{i}\right), i=1,2, \ldots, n$, be a sequence of positive real numbers. In [12], the following inequality was proven

$$
\left(\sum_{i=1}^{n} \sqrt{a_{i}}\right)^{2} \leq(n-1) \sum_{i=1}^{n} a_{i}+n\left(\prod_{i=1}^{n} a_{i}\right)^{\frac{1}{n}} .
$$

Equality in (1) holds if and only if $a_{1}=a_{2}=\cdots=a_{n}$.

If $a=\left(a_{i}\right), i=1,2, \ldots, n$, is a sequence of positive real numbers with the property $a_{1} \geq a_{2} \geq \cdots \geq a_{n}$ then it holds [4] that

$$
\sum_{i=1}^{n} a_{i} \geq n\left(\prod_{i=1}^{n} a_{i}\right)^{\frac{1}{n}}+\left(\sqrt{a_{1}}-\sqrt{a_{n}}\right)^{2}
$$

with equality if and only if $a_{2}=\cdots=a_{n-1}=\sqrt{a_{1} a_{n}}$.

Let $p=\left(p_{i}\right), i=1,2, \ldots, n$, be a sequence of non-negative real numbers, and let $a=\left(a_{i}\right), i=1,2, \ldots, n$, be a sequence of positive real numbers. Then, for any real $r$ with $r \leq 0$ or $r \geq 1$, it holds [10] (see also [15]) that

$$
\left(\sum_{i=1}^{n} p_{i}\right)^{r-1} \sum_{i=1}^{n} p_{i} a_{i}^{r} \geq\left(\sum_{i=1}^{n} p_{i} a_{i}\right)^{r}
$$

When $0 \leq r \leq 1$, the opposite inequality in (3) is valid. Equality sign in (3) holds if and only if $r=0$, or $r=1$, or $a_{1}=a_{2}=\cdots=a_{n}$, or $p_{1}=p_{2}=\cdots=p_{t}=0$ and $a_{t+1}=\cdots=a_{n}$ for some $t$ satisfying $1 \leq t \leq n-1$.

Let $a=\left(a_{i}\right)$ and $b=\left(b_{i}\right), i=1,2, \ldots, n$, be two real number sequences with the properties $0<r_{1} \leq a_{i} \leq R_{1}$ and $0<r_{2} \leq b_{i} \leq R_{2}$. In [2] (see also [16]), the following inequality was proven

$$
\left|n \sum_{i=1}^{n} a_{i} b_{i}-\sum_{i=1}^{n} a_{i} \sum_{i=1}^{n} b_{i}\right| \leq\left(R_{1}-r_{1}\right)\left(R_{2}-r_{2}\right) n\left[\frac{n}{2}\right]\left(1-\frac{1}{n}\left[\frac{n}{2}\right]\right) .
$$

Equality in (4) holds if and only if $a_{1}=a_{2}=\cdots=a_{n}$ or $b_{1}=b_{2}=\cdots=b_{n}$. Since

$$
\frac{1}{n}\left[\frac{n}{2}\right]\left(1-\frac{1}{n}\left[\frac{n}{2}\right]\right)= \begin{cases}\frac{1}{4} & \text { if } n \text { is even } \\ \frac{(n-1)(n+1)}{4 n^{2}} & \text { if } n \text { is odd }\end{cases}
$$


we have

$$
\alpha(n)=\frac{1}{n}\left[\frac{n}{2}\right]\left(1-\frac{1}{n}\left[\frac{n}{2}\right]\right)=\frac{1}{4}\left(1-\frac{(-1)^{n+1}+1}{2 n^{2}}\right) .
$$

Therefore, (4) can be rewritten as

$$
\left|n \sum_{i=1}^{n} a_{i} b_{i}-\sum_{i=1}^{n} a_{i} \sum_{i=1}^{n} b_{i}\right| \leq n^{2} \alpha(n)\left(R_{1}-r_{1}\right)\left(R_{2}-r_{2}\right) .
$$

Let $p=\left(p_{i}\right)$ and $a=\left(a_{i}\right), i=1,2, \ldots, n$, be positive real number sequences such that $a=\left(a_{i}\right)$ is monotone and $0<r \leq a_{i} \leq R<+\infty$. Let $S$ be a subset of $I_{n}=\{1,2, \ldots, n\}$ which minimizes the expression

$$
\left|\sum_{i \in S} p_{i}-\frac{1}{2} \sum_{i=1}^{n} p_{i}\right|
$$

In [14], it was proven that

$$
\sum_{i=1}^{n} p_{i} a_{i} \sum_{i=1}^{n} \frac{p_{i}}{a_{i}} \leq\left(1+\gamma(S) \frac{(R-r)^{2}}{r R}\right)\left(\sum_{i=1}^{n} p_{i}\right)^{2},
$$

where

$$
\gamma(S)=\frac{\sum_{i \in S} p_{i}}{\sum_{i=1}^{n} p_{i}}\left(\begin{array}{r}
\sum_{i \in S} p_{i} \\
\sum_{i=1}^{n} p_{i}
\end{array} .\right.
$$

Equality in (8) is attained if $R=a_{1}=\cdots=a_{n}=r$.

\section{Main results}

In this section, by making use of the inequalities specified in Section 2 we derive inequalities involving $S E I_{a}(G)$ or/and $\overline{S E I}_{a}(G)$. Firstly, we establish a lower bound and an upper bound on $S E I_{a}(G)$ in terms of the parameters $n, m, \Delta, \delta$, det $D$, and $a$ for $a>1$.

Theorem 1. If $G$ is a connected graph of order $n \geq 2$, size $m$, minimum degree $\delta$, maximum degree $\Delta$, and if the determinant of its diagonal degree matrix is $\operatorname{det} D$ then for any $a>1$ it holds that

$$
\left(\sqrt{\Delta a^{\Delta}}-\sqrt{\delta a^{\delta}}\right)^{2} \leq S E I_{a}(G)-n a^{\frac{2 m}{n}}(\operatorname{det} D)^{\frac{1}{n}} \leq n^{2} \alpha(n)\left(\sqrt{\Delta a^{\Delta}}-\sqrt{\delta a^{\delta}}\right)^{2},
$$

with equalities if and only if $G$ is a regular graph, where $\alpha(n)$ is defined via (5). 
Proof. Let $\left(d_{1}, d_{2}, \cdots, d_{n}\right)$ be the vertex-degree sequence of $G$ such that $\Delta=d_{1} \geq d_{2} \geq$ $\cdots \geq d_{n}=\delta>0$. For $a_{i}=b_{i}=\sqrt{d_{i} a^{d_{i}}}, i=1,2, \ldots, n, R_{1}=R_{2}=\sqrt{\Delta a^{\Delta}}, r_{1}=r_{2}=$ $\sqrt{\delta a^{\delta}}$, the inequality (6) transforms into

$$
0 \leq n \sum_{i=1}^{n} d_{i} a^{d_{i}}-\left(\sum_{i=1}^{n} \sqrt{d_{i} a^{d_{i}}}\right)^{2} \leq n^{2} \alpha(n)\left(\sqrt{\Delta a^{\Delta}}-\sqrt{\delta a^{\delta}}\right)^{2}
$$

that is

$$
n S E I_{a}(G) \leq\left(\sum_{i=1}^{n} \sqrt{d_{i} a^{d_{i}}}\right)^{2}+n^{2} \alpha(n)\left(\sqrt{\Delta a^{\Delta}}-\sqrt{\delta a^{\delta}}\right)^{2} .
$$

On the other hand, for $a_{i}=d_{i} a^{d_{i}}, i=1,2, \ldots, n$, the inequality (1) becomes

$$
\left(\sum_{i=1}^{n} \sqrt{d_{i} a^{d_{i}}}\right)^{2} \leq(n-1) \sum_{i=1}^{n} d_{i} a^{d_{i}}+n\left(\prod_{i=1}^{n} d_{i} a^{d_{i}}\right)^{\frac{1}{n}},
$$

i.e.,

$$
\left(\sum_{i=1}^{n} \sqrt{d_{i} a^{d_{i}}}\right)^{2} \leq(n-1) S E I_{a}(G)+n a^{\frac{2 m}{n}}(\operatorname{det} D)^{\frac{1}{n}} .
$$

From (11) and (12) we arrive at the right-hand side of (10).

Equalities in (11) and (12) hold if and only if $d_{1} a^{d_{1}}=\cdots=d_{n} a^{d_{n}}$, which implies that equality in the right-hand side of (10) holds if and only if $G$ is regular.

Now, setting $a_{i}=d_{i} a^{d_{i}}, i=1,2, \ldots, n$, in (2), we get

$$
\sum_{i=1}^{n} d_{i} a^{d_{i}} \geq n\left(\prod_{i=1}^{n} d_{i} a^{d_{i}}\right)^{\frac{1}{n}}+\left(\sqrt{\Delta a^{\Delta}}-\sqrt{\delta a^{\delta}}\right)^{2},
$$

i.e.

$$
S E I_{a}(G) \geq n a^{\frac{2 m}{n}}(\operatorname{det} D)^{\frac{1}{n}}+\left(\sqrt{\Delta a^{\Delta}}-\sqrt{\delta a^{\delta}}\right)^{2},
$$

from which we obtain the left-hand side of (10).

Equality in (13) holds if and only if $d_{2} a^{d_{2}}=\cdots=d_{n-1} a^{d_{n-1}}=\sqrt{\Delta \delta a^{\Delta+\delta}}$, that is, if and only if $\sqrt{\Delta \delta}=\frac{\Delta+\delta}{2}, \Delta=\delta$. This implies that equality in the left-hand side of (10) holds if and only if $G$ is regular.

As $\left(\sqrt{\Delta a^{\Delta}}-\sqrt{\delta a^{\delta}}\right)^{2} \geq 0$ and $\alpha(n) \leq \frac{1}{4}$, the next two results follow from Theorem 1.

Corollary 1. If $G$ is a connected graph having the parameters mentioned in the statement of Theorem 1 then for any $a>1$ it holds that

$$
n a^{\frac{2 m}{n}}(\operatorname{det} D)^{\frac{1}{n}} \leq S E I_{a}(G) \leq n a^{\frac{2 m}{n}}(\operatorname{det} D)^{\frac{1}{n}}+\frac{n^{2}}{4}\left(\sqrt{\Delta a^{\Delta}}-\sqrt{\delta a^{\delta}}\right)^{2},
$$

with equality if and only if $G$ is regular. 
Corollary 2. If $U$ is a connected unicyclic graph of order $n \geq 3$, minimum degree $\delta$, maximum degree $\Delta$, and if the determinant of its diagonal degree matrix is $\operatorname{det} D$ then for any $a>1$ it holds that

$$
\left(\sqrt{\Delta a^{\Delta}}-\sqrt{\delta a^{\delta}}\right)^{2} \leq S E I_{a}(U)-n a^{2}(\operatorname{det} D)^{\frac{1}{n}} \leq n^{2} \alpha(n)\left(\sqrt{\Delta a^{\Delta}}-\sqrt{\delta a^{\delta}}\right)^{2} .
$$

with equalities if and only if $U \cong C_{n}$, where $\alpha(n)$ is defined via (5).

Next, we prove an inequality involving $S E I_{a}(G)$ and $S E I_{a}(G)^{-1}$.

Theorem 2. If $G$ is a connected graph of size $m \geq 1$, minimum degree $\delta$, maximum degree $\Delta$, and if $a>0$ with $a \neq 1$, then it holds that

$$
S E I_{a}(G)+4 m^{2} a^{\Delta+\delta} S E I_{a}(G)^{-1} \leq 2 m\left(a^{\Delta}+a^{\delta}\right),
$$

with equality if and only if $G$ is regular.

Proof. Let $\left(d_{1}, d_{2}, \cdots, d_{n}\right)$ be the vertex-degree sequence of $G$ such that $\Delta=d_{1} \geq d_{2} \geq$ $\cdots \geq d_{n}=\delta>0$. Clearly, for every $i \in\{1,2, \ldots, n\}$, it holds that $\left(a^{\Delta}-a^{d_{i}}\right)\left(a^{\delta}-a^{d_{i}}\right) \leq 0$, which gives $a^{2 d_{i}}+a^{\Delta+\delta} \leq\left(a^{\Delta}+a^{\delta}\right) a^{d_{i}}$, and hence we have

$$
a^{d_{i}}+\frac{a^{\Delta+\delta}}{a^{d_{i}}} \leq a^{\Delta}+a^{\delta} .
$$

After multiplying (15) with $d_{i}$ and then summing over $i$ for $i=1,2, \ldots, n$, we get

$$
S E I_{a}(G)+a^{\Delta+\delta} \sum_{i=1}^{n} d_{i} a^{-d_{i}} \leq 2 m\left(a^{\Delta}+a^{\delta}\right) .
$$

On the other hand, for $r=-1, p_{i}=d_{i}, a_{i}=a^{d_{i}}$, with $i=1,2, \ldots, n,(3)$ gives

$$
\left(\sum_{i=1}^{n} d_{i}\right)^{-2} \sum_{i=1}^{n} d_{i} a^{-d_{i}} \geq\left(\sum_{i=1}^{n} d_{i} a^{d_{i}}\right)^{-1},
$$

i.e.,

$$
\sum_{i=1}^{n} d_{i} a^{-d_{i}} \geq \frac{4 m^{2}}{S E I_{a}(G)} .
$$

From (16) and (17), we arrive at (14).

Equality in (15) is attained if and only if $a^{d_{i}} \in\left\{a^{\delta}, a^{\Delta}\right\}$ for every $i=1,2, \ldots, n$. Equality in (17) holds if and only if $a^{\Delta}=a^{d_{1}}=\cdots=a^{d_{n}}=a^{\delta}$, i.e. $\Delta=d_{1}=\cdots=d_{n}=\delta$. Therefore we conclude that equality in (14) holds if and only if $G$ is regular. 
Corollary 3. If $U$ is a connected unicyclic graph of order $n \geq 3$, minimum degree $\delta$, maximum degree $\Delta$, and if $a>0$ with $a \neq 1$, then it holds that

$$
S E I_{a}(U)+4 n^{2} a^{\Delta+\delta} S E I_{a}(U)^{-1} \leq 2 n\left(a^{\Delta}+a^{\delta}\right),
$$

with equality if and only if $U \cong C_{n}$.

Let $\chi$ and $\bar{\chi}$ be the chromatic numbers of $G$ and $\bar{G}$, respectively. Nordhaus and Gaddum [17] reported bounds on $\chi \cdot \bar{\chi}$ and $\chi+\bar{\chi}$. Motivated by these results of Nordhaus and Gaddum [17], next we prove an inequality involving $S E I_{a}(G)$ and $S E I_{a}(\bar{G})$.

Theorem 3. If $G$ is a connected graph of order $n \geq 2$, size $m$, minimum degree $\delta$, maximum degree $\Delta$, and if $a>0$ with $a \neq 1$, then it holds that

$$
a^{\Delta+\delta-n+1} S E I_{a}(\bar{G})-S E I_{a}(G) \leq\left(a^{\Delta}+a^{\delta}\right)(n(n-1)-2 m)-n(n-1) a^{\frac{2 m}{n}},
$$

with equality if and only if $G$ is regular, where $\bar{G}$ is the complement of $G$.

Proof. Let $\left(d_{1}, d_{2}, \cdots, d_{n}\right)$ be the vertex-degree sequence of $G$ such that $\Delta=d_{1} \geq d_{2} \geq$ $\cdots \geq d_{n}=\delta>0$. After multiplying the inequality (15) with $n-1-d_{i}$ and summing over $i$ for $i=1,2, \ldots, n$, we get

$$
\sum_{i=1}^{n}\left(n-1-d_{i}\right) a^{d_{i}}+a^{\Delta+\delta} \sum_{i=1}^{n}\left(n-1-d_{i}\right) a^{-d_{i}} \leq\left(a^{\Delta}+a^{\delta}\right) \sum_{i=1}^{n}\left(n-1-d_{i}\right),
$$

which gives

$$
(n-1) \sum_{i=1}^{n} a^{d_{i}}-S E I_{a}(G)+a^{\Delta+\delta-n+1} S E I_{a}(\bar{G}) \leq\left(a^{\Delta}+a^{\delta}\right)(n(n-1)-2 m) .
$$

Now, by arithmetic-geometric mean inequality, AM-GM (see e.g. [15]), we have

$$
\sum_{i=1}^{n} a^{d_{i}} \geq n\left(\prod_{i=1}^{n} a^{d_{i}}\right)^{\frac{1}{n}}=n a^{\frac{2 m}{n}}
$$

From (19) and (20), we arrive at (18).

Equality in (15) is attained if and only if $a^{d_{i}} \in\left\{a^{\delta}, a^{\Delta}\right\}$ for every $i, i=1,2, \ldots, n$. Equality in (20) holds if and only if $a^{d_{1}}=\cdots=a^{d_{n}}$. Therefore, we conclude that equality in (18) holds if and only if $G$ is a regular graph.

Corollary 4. Let $G$ be a connected graph of order $n \geq 3$, size $m$, minimum degree $\delta$ and maximum degree $\Delta$. If $\bar{G}$ (complement of $G$ ) is connected and if $a>0$ with $a \neq 1$, then it holds that

$$
\overline{S E I}_{a}(G)+a^{\Delta+\delta-n+1} S E I_{a}(\bar{G}) \leq\left(a^{\Delta}+a^{\delta}\right)(n(n-1)-2 m),
$$

with equality if and only if every vertex of $G$ has degree either $\Delta$ or $\delta$. 
Theorem 4. Let $G$ be a connected graph of order $n \geq 2$ and size $m$. Let $\left(d_{1}, d_{2}, \cdots, d_{n}\right)$ be the vertex-degree sequence of $G$ such that $\Delta=d_{1} \geq d_{2} \geq \cdots \geq d_{n}=\delta>0$. For any $a>1$, it holds that

$$
S E I_{a}(G) \leq \frac{4 m^{2} a^{\frac{2 m}{n}}}{n(\operatorname{det} D)^{\frac{1}{n}}}\left(1+\gamma(S) \frac{\left(a^{\Delta}-a^{\delta}\right)^{2}}{a^{\Delta+\delta}}\right),
$$

where $\operatorname{det} D$ is the determinant of the diagonal degree matrix of $G$,

$$
\gamma(S)=\frac{\sum_{i \in S} d_{i}}{2 m}\left(1-\frac{\sum_{i \in S} d_{i}}{2 m}\right)
$$

and $S$ is a subset of $I_{n}=\{1,2, \ldots, n\}$ which minimizes the expression

$$
\left|\sum_{i \in S} d_{i}-m\right|
$$

Equality sign in (21) holds if $G$ is regular.

Proof. For $p_{i}=d_{i}$ with $i=1,2, \ldots, n,(7)$ becomes (23). Let $S$ be a subset of $I_{n}=$ $\{1,2, \ldots, n\}$ which minimizes the expression (23). Then, from (9) we get $\gamma(S)$ defined by (22). Also, for $p_{i}=d_{i}$ and $a_{i}=a^{d_{i}}$ with $i=1,2, \ldots, n$, and for $a>1, R=a^{\Delta}, r=a^{\delta}$, the inequality (8) transforms into

$$
\sum_{i=1}^{n} d_{i} a^{d_{i}} \sum_{i=1}^{n} d_{i} a^{-d_{i}} \leq\left(1+\gamma(S) \frac{\left(a^{\Delta}-a^{\delta}\right)^{2}}{a^{\Delta+\delta}}\right) 4 m^{2} .
$$

On the other hand, according to the arithmetic-geometric mean inequality, we have

$$
\sum_{i=1}^{n} d_{i} a^{-d_{i}} \geq n\left(\prod_{i=1}^{n} d_{i} a^{-d_{i}}\right)^{\frac{1}{n}}
$$

that is,

$$
\sum_{i=1}^{n} d_{i} a^{-d_{i}} \geq \frac{n(\operatorname{det} D)^{\frac{1}{n}}}{a^{\frac{2 m}{n}}} .
$$

Now, from (24) and (25) we obtain (21).

Equality in (24) holds if $G$ is a regular graph. In that case the equality in (15) is attained also. Therefore, we conclude that equality in (21) holds if $G$ is regular.

Since for any $S \subset I_{n}=\{1,2, \ldots, n\}$, it holds $\gamma(S) \leq \frac{1}{4}$, we have the following corollaries of Theorem 4 . 
Corollary 5. If $G$ is a connected graph of order $n \geq 2$, size $m$, minimum degree $\delta$, maximum degree $\Delta$, and if the determinant of its diagonal degree matrix is $\operatorname{det} D$ then for any $a>1$ it holds that

with equality if $G$ is regular.

$$
S E I_{a}(G) \leq \frac{m^{2} a^{\frac{2 m}{n}}\left(a^{\Delta}+a^{\delta}\right)^{2}}{n a^{\Delta+\delta}(\operatorname{det} D)^{\frac{1}{n}}}
$$

Corollary 6. If $U$ is a connected unicyclic graph of order $n \geq 3$, minimum degree $\delta$, maximum degree $\Delta$, and if the determinant of its diagonal degree matrix is $\operatorname{det} D$ then for any $a>1$ it holds that

$$
S E I_{a}(U) \leq \frac{n a^{2}\left(a^{\Delta}+a^{\delta}\right)^{2}}{a^{\Delta+\delta}(\operatorname{det} D)^{\frac{1}{n}}}
$$

with equality if $U \cong C_{n}$.

Theorem 5. Let $G$ be a connected graph of order $n \geq 2$, size $m$, minimum degree $\delta$ and maximum degree $\Delta$. If $a>1$ then

$$
\begin{aligned}
& (n-1)\left(n a^{\frac{2 m}{n}}+\left(\sqrt{a^{\Delta}}-\sqrt{a^{\delta}}\right)^{2}\right) \leq S E I_{a}(G)+\overline{S E I}_{a}(G) \\
& \leq n(n-1)\left(a^{\frac{2 m}{n}}+n \alpha(n)\left(\sqrt{a^{\Delta}}-\sqrt{a^{\delta}}\right)^{2}\right),
\end{aligned}
$$

and if $0<a<1$ then

$$
\begin{aligned}
& (n-1)\left(n a^{\frac{2 m}{n}}+\frac{\left(\sqrt{a^{\Delta}}-\sqrt{a^{\delta}}\right)^{2}}{a^{\Delta+\delta}}\right) \leq S E I_{a}(G)+\overline{S E I}_{a}(G) \\
& \leq n(n-1)\left(a^{\frac{2 m}{n}}+\frac{n \alpha(n)\left(\sqrt{a^{\Delta}}-\sqrt{a^{\delta}}\right)^{2}}{a^{\Delta+\delta}}\right)
\end{aligned}
$$

where $\alpha(n)$ is defined via (5). Left equality signs in (26) and (27) hold if $d_{2}=\cdots=$ $d_{n-1}=\frac{\Delta+\delta}{2}$. Right equality sign in (26) and (27) hold if and only if $G$ is regular.

Proof. Let $\left(d_{1}, d_{2}, \cdots, d_{n}\right)$ be the vertex-degree sequence of $G$ such that $\Delta=d_{1} \geq d_{2} \geq$ $\cdots \geq d_{n}=\delta>0$. For any $a>0$ with $a \neq 1$, the following identity is valid

$$
S E I_{a}(G)+\overline{S E I}_{a}(G)=(n-1) \sum_{i=1}^{n} a^{d_{i}} .
$$

First, we assume that $a>1$. For $a_{i}=b_{i}=\sqrt{a^{d_{i}}}$ with $i=1,2, \ldots, n$, and $R_{1}=R_{2}=\sqrt{a^{\Delta}}$, $r_{1}=r_{2}=\sqrt{a^{\delta}}$, the inequality (6) transforms into

$$
0 \leq n \sum_{i=1}^{n} a^{d_{i}}-\left(\sum_{i=1}^{n} \sqrt{a^{d_{i}}}\right)^{2} \leq n^{2} \alpha(n)\left(\sqrt{a^{\Delta}}-\sqrt{a^{\delta}}\right)^{2}
$$


that is

$$
n \sum_{i=1}^{n} a^{d_{i}} \leq\left(\sum_{i=1}^{n} \sqrt{a^{d_{i}}}\right)^{2}+n^{2} \alpha(n)\left(\sqrt{a^{\Delta}}-\sqrt{a^{\delta}}\right)^{2} .
$$

Now, setting $a_{i}=a^{d_{i}}$ with $i=1,2, \ldots, n$, in (1), we get

$$
\left(\sum_{i=1}^{n} \sqrt{a^{d_{i}}}\right)^{2} \leq(n-1) \sum_{i=1}^{n} a^{d_{i}}+n a^{\frac{2 m}{n}} .
$$

From (29) and (30), it follows that

$$
n \sum_{i=1}^{n} a^{d_{i}} \leq(n-1) \sum_{i=1}^{n} a^{d_{i}}+n a^{\frac{2 m}{n}}+n^{2} \alpha(n)\left(\sqrt{a^{\Delta}}-\sqrt{a^{\delta}}\right)^{2},
$$

that is

$$
\sum_{i=1}^{n} a^{d_{i}} \leq n a^{\frac{2 m}{n}}+n^{2} \alpha(n)\left(\sqrt{a^{\Delta}}-\sqrt{a^{\delta}}\right)^{2} .
$$

From (28) and (31), we obtain the right-hand side of (26).

Equalities in (29) and (30) hold if and only if $a^{d_{1}}=a^{d_{2}}=\cdots=a^{d_{n}}$, therefore equality in the right-hand side of (26) holds if and only if $G$ is regular.

Now, for $a_{i}=a^{d_{i}}$ with $i=1,2, \ldots, n$, the inequality (2) becomes

$$
\sum_{i=1}^{n} a^{d_{i}} \geq n a^{\frac{2 m}{n}}+\left(\sqrt{a^{\Delta}}-\sqrt{a^{\delta}}\right)^{2} .
$$

From (28) and (32), we get the left-hand side of (26).

Equality in (32) holds if $a^{d_{2}}=\cdots=a^{d_{n-1}}=a^{\frac{\Delta+\delta}{2}}$, therefore equality in the left-hand side of (26) holds if $d_{2}=\cdots=d_{n-1}=\frac{\Delta+\delta}{2}$.

In a similar way, one can prove that $(27)$ holds for $0<a<1$.

Since $\alpha(n) \leq \frac{1}{4}$, the next result is a direct consequence of Theorem 5 .

Corollary 7. Let $G$ be a connected graph of order $n \geq 2$, size $m$, minimum degree $\delta$ and maximum degree $\Delta$. If $a>1$ then

$$
S E I_{a}(G)+\overline{S E I}_{a}(G) \leq n(n-1)\left(a^{\frac{2 m}{n}}+\frac{n\left(\sqrt{a^{\Delta}}-\sqrt{a^{\delta}}\right)^{2}}{4}\right),
$$

and if $0<a<1$ then

$$
S E I_{a}(G)+\overline{S E I}_{a}(G) \leq n(n-1)\left(a^{\frac{2 m}{n}}+\frac{n\left(\sqrt{a^{\Delta}}-\sqrt{a^{\delta}}\right)^{2}}{4 a^{\Delta+\delta}}\right),
$$

where the equalities hold if and only if $G$ is regular. 
For unicyclic graphs, we have the following two corollaries of Theorem 5.

Corollary 8. Let $U$ be a connected unicyclic graph of order $n \geq 3$, minimum degree $\delta$ and maximum degree $\Delta$. If $a>1$ then

$$
\begin{aligned}
& (n-1)\left(n a^{2}+\left(\sqrt{a^{\Delta}}-\sqrt{a^{\delta}}\right)^{2}\right) \leq S E I_{a}(U)+\overline{S E I}_{a}(U) \\
& \leq n(n-1)\left(a^{2}+n \alpha(n)\left(\sqrt{a^{\Delta}}-\sqrt{a^{\delta}}\right)^{2}\right)
\end{aligned}
$$

and if $0<a<1$ then

$$
\begin{aligned}
& (n-1)\left(n a^{2}+\frac{\left(\sqrt{a^{\Delta}}-\sqrt{a^{\delta}}\right)^{2}}{a^{\Delta+\delta}}\right) \leq S E I_{a}(U)+\overline{S E I}_{a}(U) \\
& \leq n(n-1)\left(a^{2}+\frac{n \alpha(n)\left(\sqrt{a^{\Delta}}-\sqrt{a^{\delta}}\right)^{2}}{a^{\Delta+\delta}}\right),
\end{aligned}
$$

where the equality signs hold if $U \cong C_{n}$.

Corollary 9. If $U$ is a connected unicyclic graph with $n \geq 3$ vertices then for any $a>0$ with $a \neq 1$, it holds that

$$
S E I_{a}(U)+\overline{S E I}_{a}(U) \geq n(n-1) a^{2},
$$

with equality if and only if $U \cong C_{n}$.

Theorem 6. If $G$ is a connected graph with $n \geq 2$ vertices and $m$ edges then for any $a>0$ with $a \neq 1$, it holds that

$$
S E I_{a}(\bar{G}) \cdot \overline{S E I}_{a}(G) \geq a^{n-1}(n(n-1)-2 m)^{2} .
$$

Equality in (33) holds if and only if either $G$ is regular or $n-1=d_{1}=\cdots=d_{t}>d_{t+1}=$ $\cdots=d_{n}=\delta$, for some $t$ with $1 \leq t \leq n-1$.

Proof. If $G \cong K_{n}$, then obviously the equality holds in (33). In what follows, we assume that $G \not K_{n}$. Let $\left(d_{1}, d_{2}, \cdots, d_{n}\right)$ be the vertex-degree sequence of $G$ such that $\Delta=$ $d_{1} \geq d_{2} \geq \cdots \geq d_{n}=\delta>0$. For any $a>0$ with $a \neq 1$, it holds that

$$
S E I_{a}(\bar{G})=\sum_{i=1}^{n}\left(n-1-d_{i}\right) a^{n-1-d_{i}}=a^{n-1} \sum_{i=1}^{n}\left(n-1-d_{i}\right) a^{-d_{i}} .
$$


For $r=-1, p_{i}=n-1-d_{i}$ and $a_{i}=a^{d_{i}}$ with $i=1,2, \ldots, n$, the inequality (3) gives

$$
\left(\sum_{i=1}^{n}\left(n-1-d_{i}\right)\right)^{-2} \sum_{i=1}^{n}\left(n-1-d_{i}\right) a^{-d_{i}} \geq\left(\sum_{i=1}^{n}\left(n-1-d_{i}\right) a^{d_{i}}\right)^{-1},
$$

that is

$$
(n(n-1)-2 m)^{-2} \sum_{i=1}^{n}\left(n-1-d_{i}\right) a^{-d_{i}} \geq\left(\overline{S E I}_{a}(G)\right)^{-1} .
$$

From (34) and (35), we get (33).

Equality in (35), and consequently in (33), is attained if and only if either $G$ is a regular graph or $n-1=d_{1}=\cdots=d_{t}>d_{t+1}=\cdots=d_{n}=\delta$, for some $t$ with $1 \leq t \leq n-1$.

Corollary 10. If $U$ is a connected unicyclic graph with $n \geq 3$ vertices then for any $a>0$ with $a \neq 1$, it holds that

$$
S E I_{a}(\bar{U}) \cdot \overline{S E I}_{a}(U) \geq a^{n-1} n^{2}(n-3)^{2},
$$

with equality if and only if $U \cong C_{n}$.

Theorem 7. If $G$ is a connected graph with $n \geq 2$ vertices and $m$ edges then for any $a>1$, it holds that

$$
\overline{S E I}_{a}(G)+S E I_{a}(\bar{G}) \geq 2(n(n-1)-2 m) a^{\frac{n-1}{2}} .
$$

If $0<a<1$, the opposite inequality sign in (36) is valid. The equality sign in (36) holds if and only if $n$ is odd and $G$ is an $\left(\frac{n-1}{2}\right)$-regular graph.

Proof. Let $\left(d_{1}, d_{2}, \cdots, d_{n}\right)$ be the vertex-degree sequence of $G$ such that $d_{1} \geq d_{2} \geq \cdots \geq$ $d_{n}$. Here, one has

$$
\overline{S E I}_{a}(G)+S E I_{a}(\bar{G})=\sum_{i=1}^{n}\left(n-1-d_{i}\right)\left(a^{d_{i}}+a^{n-1-d_{i}}\right) .
$$

First, we assume that $a>1$. Since the function $f(x)=a^{x}$, with $x \in[1,+\infty)$, is convex, so for any $x, y \in[1,+\infty)$ we have $\frac{1}{2}\left(a^{x}+a^{y}\right) \geq a^{\frac{x+y}{2}}$ (see e.g. [16]), and thereby

$$
\frac{1}{2}\left(a^{d_{i}}+a^{n-1-d_{i}}\right) \geq a^{\frac{n-1}{2}} .
$$

From (37) and (38), we get

$$
\overline{S E I}_{a}(G)+S E I_{a}(\bar{G}) \geq 2 \sum_{i=1}^{n}\left(n-1-d_{i}\right) a^{\frac{n-1}{2}}=2(n(n-1)-2 m) a^{\frac{n-1}{2}},
$$


which completes the proof of (36).

Equality in (38) holds if and only if $d_{i}=\frac{n-1}{2}$ for $i=1,2, \ldots, n$, which implies that equality in (36) holds if and only if $n$ is odd and $G$ is an $\left(\frac{n-1}{2}\right)$-regular graph.

Similarly, we prove that the opposite inequality in (36) is valid when $0<a<1$.

Corollary 11. If $G$ be a connected graph with $n \geq 2$ vertices and $m$ edges then for any $a>1$, it holds that

$$
S E I_{a}(G)+\overline{S E I_{a}}(\bar{G}) \geq 4 m a^{\frac{n-1}{2}} .
$$

If $0<a<1$ then the opposite inequality sign in (39) holds. The equality sign in (39) holds if and only if $n$ is odd and $G$ is an $\left(\frac{n-1}{2}\right)$-regular graph.

\section{References}

[1] A. Ali, D. Dimitrov, On the extremal graphs with respect to bound incident degree indices, Discr. Appl. Math. 238 (2018) 32-40.

[2] M. Biernacki, H. Pidek, C. Ryll-Nardzewski, Sur une inegualite entre des integrales defines, Annales Univ. Mariae Curie-Skladowska A4 (1950) 1-4.

[3] W. Carballosa, J. M. Rodríguez, J. M. Sigarreta, Extremal problems on the variable sum exdeg index, MATCH Commun. Math. Comput. Chem. 84 (2020) 753-772.

[4] V. Cirtoaje, The best lower bound depended on two fixed variables for Jensen's inequality with ordered variables, J. Ineq. Appl. 2010 (2010) \#128258.

[5] D. Dimitrov, A. Ali, On the extremal graphs with respect to the variable sum exdeg index, Discr. Math. Lett. 1 (2019) 42-48.

[6] T. Došlić, Vertex-weighted Wiener polynomials for composite graphs, Ars Math. Contemp. 1 (2008) 66-80.

[7] J. Du, X. Sun, On the graph connectivity and the variable sum exdeg index, AIMS Math. 6 (2020) 607-622.

[8] A. Ghalavand, A. R. Ashrafi, Extremal graphs with respect to variable sum exdeg index via majorization, Appl. Math. Comput. 303 (2017) 19-23. 
[9] M. Javaid, A. Ali, I. Milovanović, E. Milovanović, On the extremal cactus graphs for variable sum exdeg index with a fixed number of cycles, AKCE Int. J. Graphs Combin. 17 (2020) 920-923.

[10] J. L. W. V. Jensen, Sur les fonctions convexes et les inegalites entre les valeurs moyennes, Acta Math. 30 (1906) 175-193.

[11] S. Khalid, A. Ali, On the zeroth-order general Randić index, variable sum exdeg index and trees having vertices with prescribed degree, Discr. Math. Alg. Appl. 10 (2018) \#1850015.

[12] H. Kober, On the arithmetic and geometric means and on Hölder's inequality, Proc. Am. Math. Soc. 9 (1958) 452-459.

[13] M. Matejić, E. Milovanović, I. Milovanović, A. Ali, A note on the variable sum exdeg index/coindex of trees, Contrib. Math. 2 (2020) 42-46.

[14] I. Milovanović, E. Milovanović, M. Matejić, Some inequalities for general sumconnectivity index, MATCH Commun. Math. Comput. Chem. 79 (2018) 477-489.

[15] D. S. Mitrinović, J. E. Pečarić, A. M. Fink, Classical and New Inequalities in Analysis, Kluwer Acad. Pub., Dordrecht, 1993.

[16] D. S. Mitrinović, P. M. Vasić, Analytic Inequalities, Springer, New York, 1970.

[17] E. A. Nordhaus, J. W. Gaddum, On complementary graphs, Am. Math. Monthly 63 (1956) 175-177.

[18] X. Sun, J. Du, On variable sum exdeg indices of quasi-tree graphs and unicyclic graphs, Discr. Dynam. Natur. Soc. 2020 (2020) \#1317295.

[19] B. Vukičević, Bond additive modeling 4. QSPR and QSAR studies of the variable Adriatic indices, Croat. Chem. Acta 84 (2011) 87-91.

[20] B. Vukičević, Bond additive modeling 5. Mathematical properties of the variable sum exdeg index, Croat. Chem. Acta 84 (2011) 93-101. 